\title{
Effect of Specimen Size, Compressibility and Inertia on the Response of Rigid Polymer Foams Subjected to High Velocity Direct Impact Loading
}

\author{
Behrad Koohbor ${ }^{1}$, Addis Kidane ${ }^{1 *}$, Wei-Yang Lu$^{2}$ \\ 1. Department of Mechanical Engineering, University of South Carolina, 300 Main Street, Columbia, SC 29208 \\ 2. Sandia National Laboratories, Livermore, CA 94551-0969, USA
}

\begin{abstract}
The influences of specimen length-to-diameter ratio, material compressibility, and inertia on direct impact response of high density closed-cell polymeric foam are investigated. High speed photography and stereovision digital image correlation are conducted to measure the full-field deformation response of the material subjected to direct impact. Inertia stress developed in the specimen is calculated from the acceleration distribution obtained from fullfield measurements. Total axial stress magnitude along the axis of the specimen is then reconstructed from inertia and boundary-measured stresses. It is clearly shown that there is an appreciable degree of spatial variability in strains, strain rates and stresses developed in the impacted foam specimens, whereas the degree of such axial variability is more significant at higher length-to-diameter ratios. The study is further extended to take advantage of such spatial variability to identify the rate sensitivity of the examined material over a wide range of strain rates from $1000 \mathrm{~s}^{-1}$ to $5000 \mathrm{~s}^{-1}$. The approach proposed here is shown to facilitate the identification of viscoplastic constitutive response of low impedance materials using a minimum number of tests.
\end{abstract}

\footnotetext{
Corresponding Author

Address: Department of Mechanical Engineering, University of South Carolina, 300 Main Street, Columbia, South Carolina, 29208 | Email: kidanea@cec.sc.edu | Tel: +1 (803) 7772502 | Fax: +1 (803) 7770106
} 
Keywords: polymeric foam; direct impact; digital image correlation; non-parametric analysis; constitutive response

\section{Introduction}

Rigid polymeric foams have gained extensive attention in several engineering applications that require excellent energy absorption with structural stability and significant weight reduction. Rigid polymer foams are widely used in applications such as cushioning, impact and crash mitigation, packaging, sandwich structures, etc., many of which entail high strain rate loading conditions [1]. Therefore, characterization of the mechanical response of foams under dynamic loading conditions has been an interesting subject of study for decades [2]. In this regard, several models have so far been proposed to characterize the phenomenological constitutive behavior of polymeric foams under large strain and various strain rate conditions [3-5].

From an experimental perspective, there are major challenges encountered during the study of deformation response of polymeric foams at high strain rate loading conditions [6]. The first and probably most important challenge is associated with the delayed stress equilibrium, which is due to the low mechanical impedance nature of these materials. There have been several solutions proposed to minimize the effect of such belated equilibrium conditions. For instance, application of polymeric bars [2, 7, 8] or hollow metallic transmission bar [9] in the split Hopkinson pressure bar (SHPB) experiments have been proposed with the purpose of reducing the impedance mismatch between the specimen and the bars, and hence to acquire more accurate transmitted signals. However, application of viscoelastic bars raises other challenges associated with wave propagation attenuation and dispersion due to the material rheological properties and the radial inertia stresses developed in the bars [10]. Different methods have been proposed and successfully practiced to correct the strain signals and 
restore the actual strain exerted at the interface of the specimen and the bars [11-15]. Pulse shaping technique has been established to provide an alternative solution in dynamic testing of low impedance materials. Application of pulse shaper has been established to ensure nearly constant strain rates, as well as the presence of dynamically equilibrated stress in the specimen $[16,17]$.

A more general solution to minimize the effects of non-equilibrium stress state in dynamic testing of low impedance materials is reducing the length of the sample. In general, wave reverberation period is significantly reduced in short specimens, resulting in a faster stress equilibration within the gauge area $[18,19]$. In this regard, in the case of polymeric foams and similar cellular structures there is an additional challenge due to the representative volume element (RVE) size of the specimen, which requires a minimum number of cells to be present along the specimen length, in order to capture the continuum scale response of the material $[6,20,21]$; therefore limiting the minimum thickness of the tested specimen. None of the proposed solutions discussed above are capable of taking the local deformation, inertia and compressibility into account from an experimental perspective. Note that there are numerical simulations available in the literature that accurately describe the effects due to the aforementioned challenges on low impedance cellular materials, see e.g. [6]. However, from an experimental testing standpoint, there are still certain imitations that necessitate the development of alternative solutions to compensate for above-mentioned restriction.

Recent advances in the areas of high speed photography and full-field measurements have facilitated the study of deformation and failure of different materials in a wide range of time and length scales by accounting for the local deformation information [22-26]. In this regard, extensive attention has been drawn towards the application of virtual fields method and nonparametric approaches in dynamic deformation analysis of low impedance materials [27-31]. For instance, a non-parametric approach was proposed by Othman et al. [29] by which the 
full-field stress-strain response of low impedance synthetic rubber specimens was retrieved. This approach is based on the direct calculation of local stress magnitude using the inertia stress computed from acceleration field, and the boundary measured stress. This nonparametric method was successfully implemented by the authors of the present work to characterize dynamic deformation behavior of compressible polymeric foams under direct impact and at strain rates of up to $2600 \mathrm{~s}^{-1}[30,31]$.

The main objective in this paper is to extend the previous works further by investigating the concurrent effects of specimen aspect ratio and the associated inertia effects and compressibility on the mechanical response of polymeric foams subjected to direct impact loading. Specimens with two different length-to-diameter ratios are subjected to direct impact loading using a modified shock tube apparatus. Deformation of the specimen is measured insitu using high speed photography and digital image correlation. Using a non-parametric analysis along with displacement and acceleration fields acquired from image correlation, local stress-strain curves along specimen axis are extracted. It is observed that the transient deformation state creates a high variability in the local strain rate response over the length of the specimen. Local stress-strain curves are extracted at several positions along the specimen axis, each position with a different strain rate history. Attempts are made to utilize the entire set of constitutive data to extract useful stress-strain responses for the material over a wide range of strain rate conditions using a minimum number of impact tests. This was originally achieved by Avril et al. [32] to identify elasto-visco-plastic constitutive response using a single test at quasi-static loading conditions. Accordingly, the present work attempts to investigate the feasibility of extracting meaningful rate-dependent constitutive models from a single experiment carried out at very high strain rate conditions. The method is validated by comparing the results obtained from the proposed approach with those obtained from conventional SHPB tests. 


\section{Experimental Procedure}

\subsection{Material and Specimen Geometry}

The material examined in this work is a rigid closed-cell polyurethane foam supplied by Sandia National Laboratories, under the commercial name TufFoam35. Nominal density of the as-received stock was measured as $560 \mathrm{~kg} / \mathrm{m}^{3}$ (35 pcf). Cellular structure of the foam is presented in Fig. 1a, indicating average pore dimensions of $150 \mu \mathrm{m}$ and $120 \mu \mathrm{m}$ cell-wall thickness. Compressive elastic modulus of the material was determined as the initial slope of the quasi-static stress-strain curves averaged from three independent measurements and found to be $(780 \pm 20 \mathrm{MPa})$.Longitudinal wave speed of the examined foam, $c_{l}$, was calculated from the initial density and compressive elastic modulus and found to be $1180 \mathrm{~m} / \mathrm{s}$,.

Cylindrical specimens with two different length-to-diameter ratios are extracted from the asreceived billets, the dimensions of which are shown in Fig. 1b. Specimens are extracted from a single billet using a $\mathrm{CNC}$ waterjet, with a relatively smooth lateral surface finish and a \pm 0.1 $\mathrm{mm}$ dimensional variability. For image correlation purposes, a high contrast speckle pattern is applied on the lateral surface of each specimen. The speckle pattern consists of random black and white particles applied using conventional airbrush. Average speckle size is $100 \mu \mathrm{m}$.

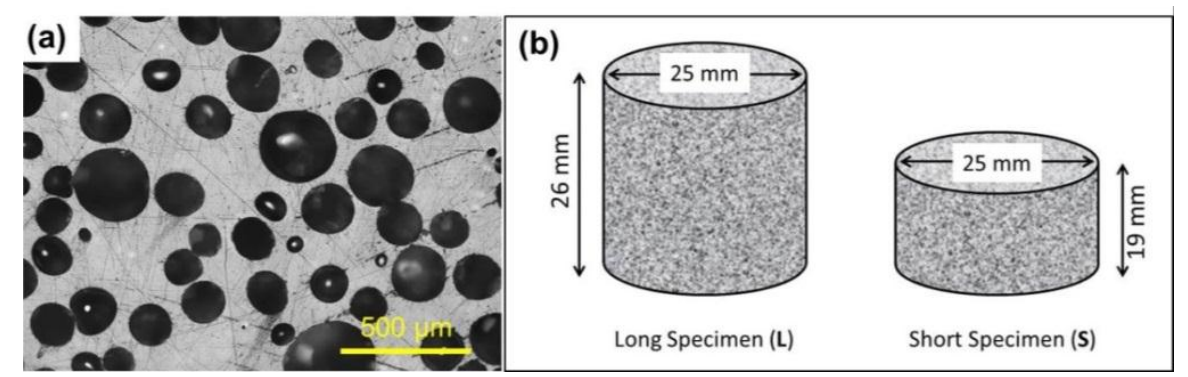


Fig. 1- (a) Cellular structure of the foam examined in this work. (b) Cylindrical specimens with different initial dimensions used for direct impact experiments.

\subsection{Impact Loading}

A modified shock tube apparatus is utilized to apply controlled direct impact on the specimens in the present work. Details on the design features of our shock tube can be found elsewhere $[24,30]$. The shock tube is schematically shown in Fig. 2a. Driver and driven sections of the tube are separated by stretched plastic diaphragms, under the commercial name "Mylar" sheets. The speckled specimen is fixed at the muzzle of the tube, inserted on a custom made load fixture. The specimen is simply supported by the load cell in one end and free on the projectile side. To keep the specimen intact with the load cell, a lithium grease is used, which also serves as a lubricant to minimize the effect of friction. The other end of the specimen along the impactor side is free, but the same lubricant is applied to reduce the effects of frictional stresses on the impacted end of the specimen. Further details on the load fixture utilized in this work can be found in $[30,31] .$. The applied dynamic load is measured on the back side of the specimen using a piezoelectric load-cell, as shown in Fig. 2b. The load-cell used in this work is $88.8 \mathrm{kN}$ capacity PCB piezotronics ${ }^{\circledR}$ load-cell with $56 \mathrm{mV} / \mathrm{kN}$ sensitivity, designed primarily to measure compressive and impact forces. A polyimide film tap covering the cap surface of the load-cell reduces the effect of high frequency ringing associated with metal-to-metal contact.

To increase the momentum transferred to the specimen and achieve higher strain rates, a high strength 70 gr aluminum projectile is directly shot at the specimen. The velocity of the projectile at the instant of impact can be manipulated by varying the thickness of the Mylar 
diaphragms [31]. Nominal strain rate applied on the specimen in dynamic compression can be calculated as [33]:

$\dot{\varepsilon}_{\text {nom }}=\frac{v}{L_{0}}$

where $v$ and $L_{0}$ represent the impactor velocity and initial specimen length, respectively. In the present work, to facilitate the study of the effect of specimen aspect ratio, impactor velocities are selected such that the resulting $\dot{\varepsilon}_{n o m}$ will be consistent for both specimen geometries. Therefore, the impactor velocities selected for direct impact experiments are 162 $\mathrm{m} / \mathrm{s}$ and $123 \mathrm{~m} / \mathrm{s}$ for $L_{0}=26 \mathrm{~mm}$ (long) and $L_{0}=19 \mathrm{~mm}$ (short) specimens, respectively (see Fig. 1).

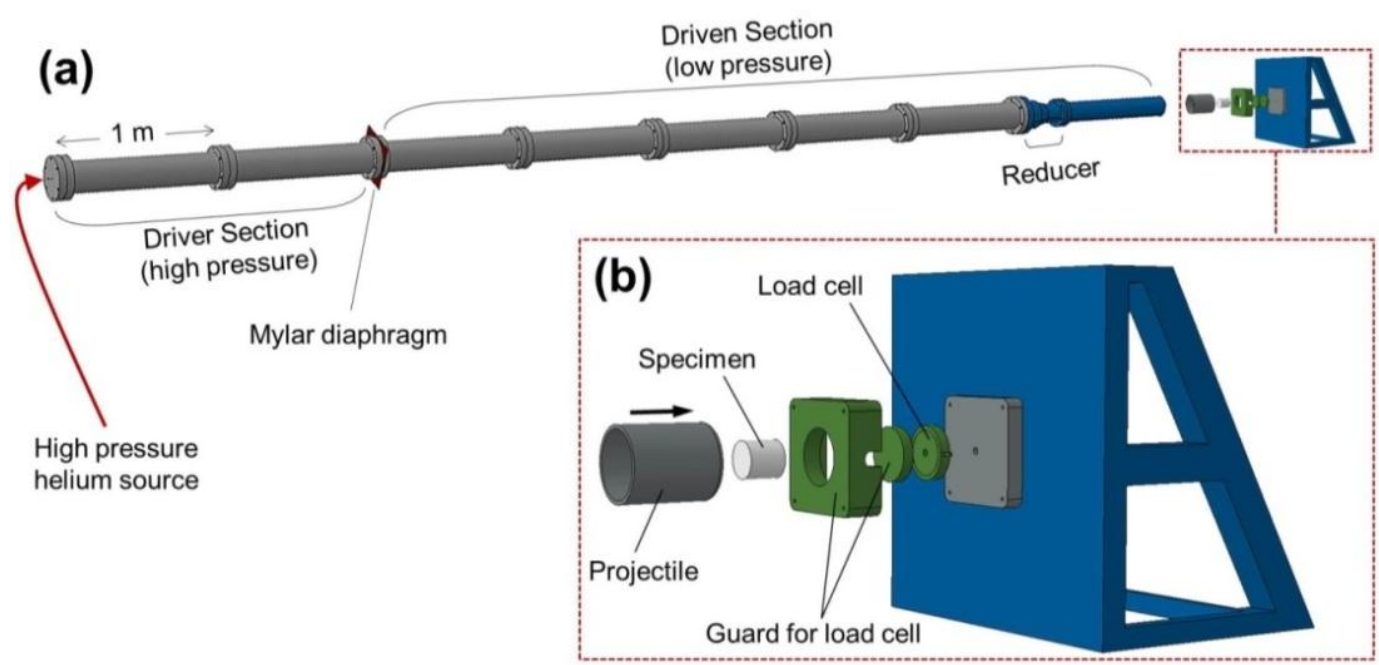

Fig. 2- (a) Schematic of the shock tube in its entirety, with a magnified view of the load fixture shown in (b)

\subsection{High Speed Imaging and Digital Image Correlation}


High speed stereo imaging in conjunction with 3D digital image correlation is used to characterize the full-field dynamic deformation response of specimens. The stereo camera system consists of two Photron Fastcam SA-X2 cameras each equipped with a $100 \mathrm{~mm}$ macro lens. $256 \times 152$ pixel $^{2}$ images are acquired at a rate of $2 \times 10^{5} \mathrm{fps}$, resulting in interframe intervals of $5 \mu \mathrm{s}$. The magnification factor is approximately $5.26 \mathrm{pixel} / \mathrm{mm}$.

Image acquisition rate is synchronized with the rate of load-cell data collection through a high speed data acquisition system and an oscilloscope. High intensity white LED lights are employed as the illumination source. Stereo-camera system is calibrated using standard calibration plates prior to the onset of impact experiments. More details on the experimental setup, stereo camera system features and the resolution of the measurements can be found in [31].

Stereo images acquired during the deformation time are analyzed in the image correlation software Vic-3D (Correlated Solutions, Inc.). Subset and step sizes of 19 pixel and 1 pixel, respectively, are used for the correlation process. Full-field strain distribution was determined using Gaussian strain filtering with a filter size of 13 . Temporal smoothing of the displacement data was conducted using $2^{\text {nd }}$ order polynomial with $10 \mu$ s time filters.

\section{Data Analysis}

The local stress as a function of length and time is extracted by accounting for inertia using Eq. 2, developed from general dynamic equilibrium equation [31].

$$
\sigma(z, t)=\sigma(0, t)+\int_{\xi=0}^{\xi=z} \rho(\xi, t) a(\xi, t) d \xi
$$


where $z=0$ is the location on which force can be measured, i.e. location of the load-cell. $\rho(\xi, t)$ is local density and $a(\xi, t)$ is axial acceleration at a given time, $t$, and given position, $\xi$.

The integral on the right hand-side of Eq. 2 represents the inertia stress, which itself is a function of the spatial variations of axial acceleration and mass density. The fundamental idea here is to evaluate the variation of density and acceleration in time and space, and use these parameters to determine the distribution of inertia stress in the material. This inertia stress can then be superimposed with the boundary measured stress, i.e. $\sigma(0, t)$, to facilitate the calculation of the total axial stress.

In cases where dynamic stress analysis of incompressible materials (e.g. metals) is to be studied, mass density will remain constant and hence can be simply taken out of the integral. However, for the case of cellular materials, such as the one considered in this work, the density changes with the applied strain. To compensate for the change of density, the analytical model shown in Eq. 3 is considered:

$\rho(z, t)=\rho_{0}\left[\exp \left(\varepsilon_{z}(z, t)\right)\right]^{2 v(z, t)-1}$

The compressibility model shown in Eq. 3 is proposed based on the principle of mass conservation and allows for the calculation of the local mass density, $\rho(z, t)$, at any given time, $t$, and location, $z$, along the specimen length, as a function of initial density, $\rho_{0}$, local axial compressive strain, $\varepsilon_{z}(z, t)$, and local Poisson's ratio of the material, $v(z, t)$. Details on the derivation of the proposed model are beyond the scope of this work, but can be found elsewhere [31]. 
Once the acceleration and density at any time and location along the specimen length are known, the inertia term can be evaluated. The integral term on the right hand-side of Eq. 2 must be evaluated over the entire area of interest. To estimate this integral, the cylindrical geometry of the specimen is first sliced into several thinner sections. The number of these thin sections depends on the number of measurement points in the length direction, which itself is a function of subset size and step size used in the image correlation process. In the present work, short and long specimens are sliced into 80 and 110 thin sections, respectively. The thickness of each section, $s_{(i)}$, is $\sim 0.22 \mathrm{~mm}$ (see Fig. 3).

Density and acceleration values within each thin section are calculated by averaging the entire population of measurement points inside each section. In this way, a more circumferentially-homogeneous distribution of each parameter is obtained (see Fig. 4). Next, the inertia stress integral in Eq. 2 is approximated as:

$\int_{z=0}^{z=L} \rho(z, t) a_{z}(z, t) d z \approx \sum_{i=1}^{n} \rho_{(i)} a_{(i)} s_{(i)}$

where, $\rho_{(i)}$ and $a_{(i)}$ represent the average values of density and axial acceleration in section i. $s_{(i)}$ is the thickness of section $i$, as depicted in Fig. 3. $n$ denotes the number of thin sections counted from left (Load-cell) side of Fig. 3 up to the location of interest. 


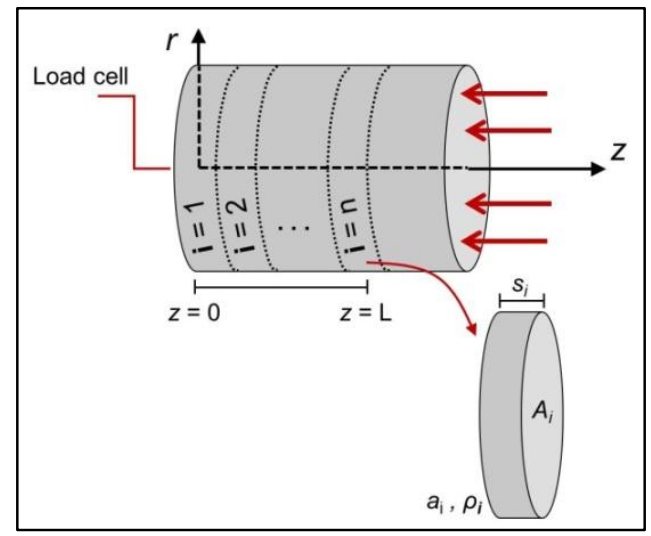

Fig. 3- Cylindrical specimen sliced into a finite number of thinner sections, used to calculate the inertia stress applied at each point along the specimen axis.

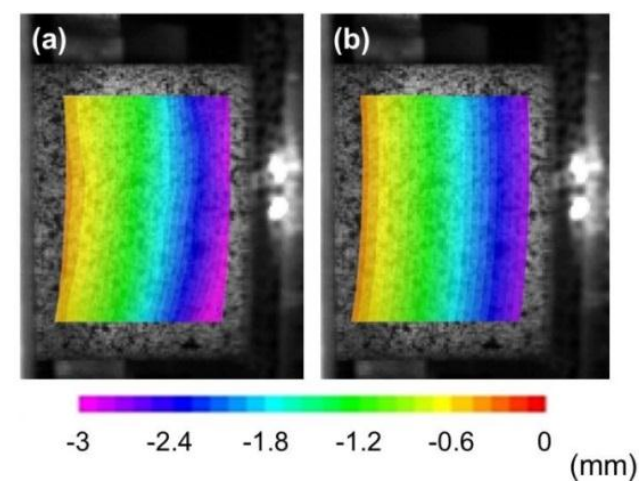

Fig. 4- Typical full-field distribution of axial displacement, (a) as obtained via DIC and (b) after sectioning and averaging over each thinner section.

Having obtained the inertia stress as a function of time, the local total stress value can be determined by superimposing the boundary-measured stress (measured at the load-cell side) with the inertia stress evaluated from Eq. 4. Local axial strain can also be readily extracted from the digital image correlation analysis. Note that the strain rate might vary spatially across the length of the specimen. Therefore, the local stress-strain responses obtained using the above procedure represent the local material response at variable strain rates. 


\section{Results and Discussion}

\subsection{Dynamic Deformation Response and Stress Equilibrium}

Fig. 5 shows the deformation sequence for both specimens at different times after the impact. The geometry of specimen after the impact indicates a mushroom shape formation at the impacted side, similar to observations made by Wang et al. [34]. Initiating from the impact side, surface failure followed by crushing are observed for short and long specimens at $t=55$ $\mu \mathrm{s}$ and $t=40 \mu \mathrm{s}$, respectively. Therefore, the whole analysis is conducted over the first $55 \mu \mathrm{s}$ for the short specimen, and $40 \mu \mathrm{s}$ for the long specimen. Videos showing the deformation and failure of the specimens are available as supplementary materials.

Based on the longitudinal elastic wave speed calculated for the foam material examined here $\left(c_{l}=1180 \mathrm{~m} / \mathrm{s}\right)$, the time for a single stress wave traverse along the specimen axis, i.e. specimen characteristic time, is calculated as $16 \mu$ s for the short specimen, and $22 \mu$ s for the long specimen. It is well-established that at least three consecutive stress wave reverberations are required for stress equilibration [35]. Considering the characteristic times calculated for the specimens in this work, a minimum time of $48 \mu$ s will be required to achieve equilibrium for the short specimen. The equilibrium condition is expected to establish after a $66 \mu$ s delay time after the impact, for the long specimen. Note that the long specimen fails well before stress equilibration, while failure in short specimen takes place few microseconds after equilibrium. Interestingly, for both cases the transient duration is comparable to the entire duration of the test, indicating that the influence of inertial loading must be carefully studied during the entire course of deformation. One particular idea in the current work is to investigate different cases where (1) failure time is comparable to the stress equilibration time, and (2) failure takes place before the establishment of quasi-static equilibrium. In such 
conditions, obtaining the material's constitutive response following conventional methods, based only on boundary measurements, is quite inadequate. This idea was justified by the use of short and long specimens.

(a)

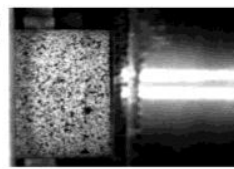

$\mathrm{t}=0 \mu \mathrm{s}$

(b)

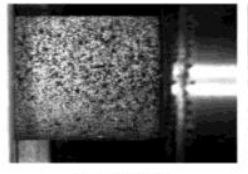

$\mathrm{t}=0 \mu \mathrm{s}$

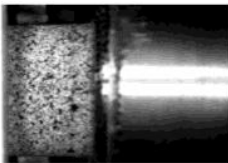

$\mathrm{t}=20 \mu \mathrm{s}$

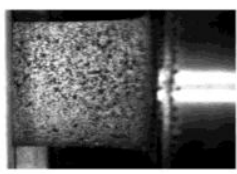

$\mathrm{t}=15 \mu \mathrm{s}$

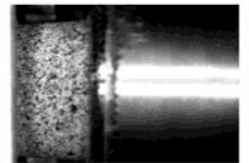

$\mathrm{t}=40 \mu \mathrm{s}$

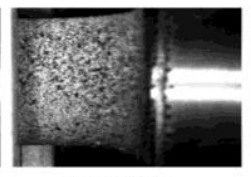

$\mathrm{t}=30 \mu \mathrm{s}$

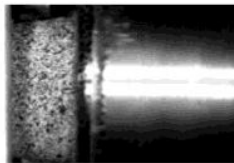

$\mathrm{t}=55 \mu \mathrm{s}$

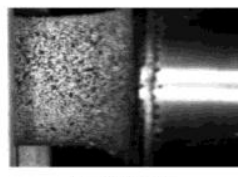

$\mathrm{t}=40 \mu \mathrm{s}$

Fig. 5- Deformation sequence at different times after the impact for (a) short and (b) long specimen. The reference time $(t=0)$ denotes the time of first contact.

\subsection{Full-Field Strain}

To investigate the deformation homogeneity in the specimens, the first step is looking the full-field strain distributions. Fig. 6 shows full-field axial strain maps obtained from DIC. As shown in Fig. 6 local strains of up to 0.2, mainly in the middle sections, are developed in both specimens before failure. In addition, strong spatial gradients in axial strain along the specimen length are observed for both geometries, whereas the degree of such spatial variability is lower for the short specimen. To explain these significant strain gradients, looking the wave propagation on the sample is important. Note that the applied impact produces two distinct waves: (1) an elastic wave traveling at a speed of $1180 \mathrm{~m} / \mathrm{s}$; (2) a plastic wave moving at a slower speed. The plastic wave traverses over the length of the specimen, reducing its velocity to zero while plastically deforming the material. The stress 
developed behind the plastic wave deforms the material axially and laterally, and forms the mushroom configuration at the impacted side of the specimen, as shown earlier in Fig. 5 [36]. Furthermore, the highest strain magnitudes are also developed at the location of the plastic wave front.

(a)
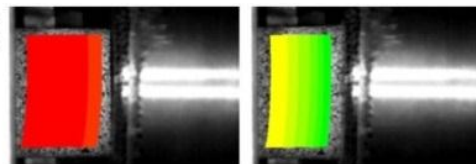

$t=20 \mu s$

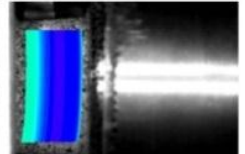

$\mathrm{t}=40 \mu \mathrm{s}$

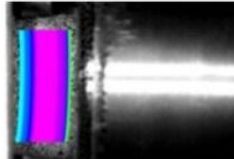

$\mathrm{t}=55 \mu \mathrm{s}$

(b)
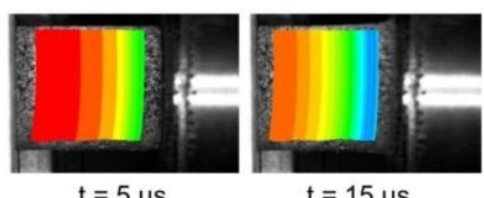

$\mathrm{t}=15 \mu \mathrm{s}$

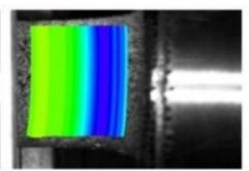

$\mathrm{t}=30 \mu \mathrm{s}$

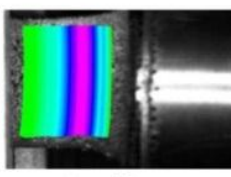

$\mathrm{t}=40 \mu \mathrm{s}$

$\begin{array}{llllll}-0.2 & -0.16 & -0.12 & -0.08 & -0.04 & 0\end{array}$

Fig. 6- Full-field axial strain for (a) short specimen and (b) long specimen.

Similar non-uniform distribution of strain was also observed in the case of radial strain component. Fig. 7 illustrates the variation of radial strain with time, extracted from five representative locations along the specimen axis. In both specimens, maximum radial strains of up to $10 \%$ are developed at the impacted side. Minimum values of radial strains are also indicated to be developed at the load-cell side. Comparing the two sets of curves shown in Figs. $7 \mathrm{~b}$ and $7 \mathrm{c}$ clearly indicates that the radial strain is distributed more inhomogeneously in the long specimen. This can be attributed to the higher impactor velocity used for the case of long specimen geometry. Note that as the contact is first established between the projectile and the specimen, waves (elastic and plastic waves) are created and travel through the specimen, changing their structure from uniaxial strain to a structure more closely associated 
with the uniaxial stress state [37]. This might be the reason behind the change of slope of the curves in Figs. $7 \mathrm{~b}$ and $7 \mathrm{c}$ at earlier deformation times $(15<t<20 \mu \mathrm{s})$ for both specimens. As the waves structure changes to the uniaxial stress, radial motion will no longer be constrained and the waves will have radial components as well as the axial ones. This change in the structure of the waves in the material results in a radial stress relief with the consequent large deformation of the material in radial direction. The magnitude of this stress relief rate is essentially a function of the impact velocity [36]. At higher impact velocities, the stress release takes place at a faster pace, giving rise to a faster radial expansion at the impacted side of the specimen, as observed in Fig. 7c.
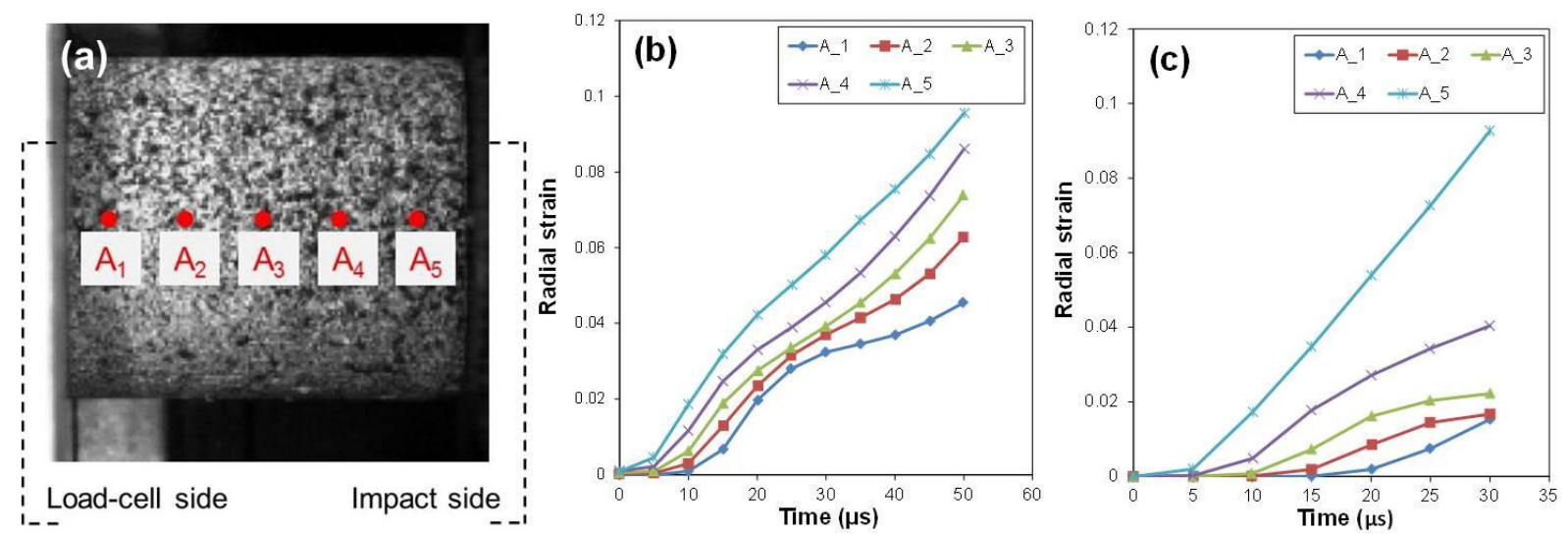

Fig. 7- Variation of radial strain components extracted from five representative points shown in (a), for (b) short and (c) long specimen.

\subsection{Poisson's Ratio and Compressibility}


Evaluation of local Poisson's ratio is essential for calculation of the material compressibility in this work (see Eq. 3). Local values of Poisson's ratio can be estimated using the full-field axial and radial strain data as:

$$
v(z, t)=-\frac{d \varepsilon_{r}(z, t)}{d \varepsilon_{z}(z, t)} \approx-\left(\frac{\varepsilon_{r}(z, t+\Delta t)-\varepsilon_{r}(z, t)}{\varepsilon_{z}(z, t+\Delta t)-\varepsilon_{z}(z, t)}\right)
$$

where $\varepsilon_{r}$ and $\varepsilon_{z}$ are the radial and axial strain components, respectively; and $\Delta t$ is the interframe time. Fig. 8 illustrates the variation of Poisson's ratio with time, for both specimen geometries.

The Poisson's ratio values depicted in Fig. 8 are simply the average of all local values over load-cell and projectile halves of the specimen. No particular trend is observed in the values determined for Poisson's ratios in this work. For both short and long specimens, the average Poisson's ratio values are higher over the regions closer to the impacted side. This observation is consistent with the fact that both specimens undergo relatively higher radial expansion on their impacted side. In addition, the $v$ values obtained for the long specimen geometry are generally lower than those of the short specimen. It should be emphasized here that that the objective of this work is not to draw any conclusive information on the Poisson's effect and its possible rate sensitivity for the utilized foam material; rather, the evolution of Poisson's ratio under direct impact loading condition is taken into account only with the purpose of facilitating the calculation of material compressibility using Eq. 3. 

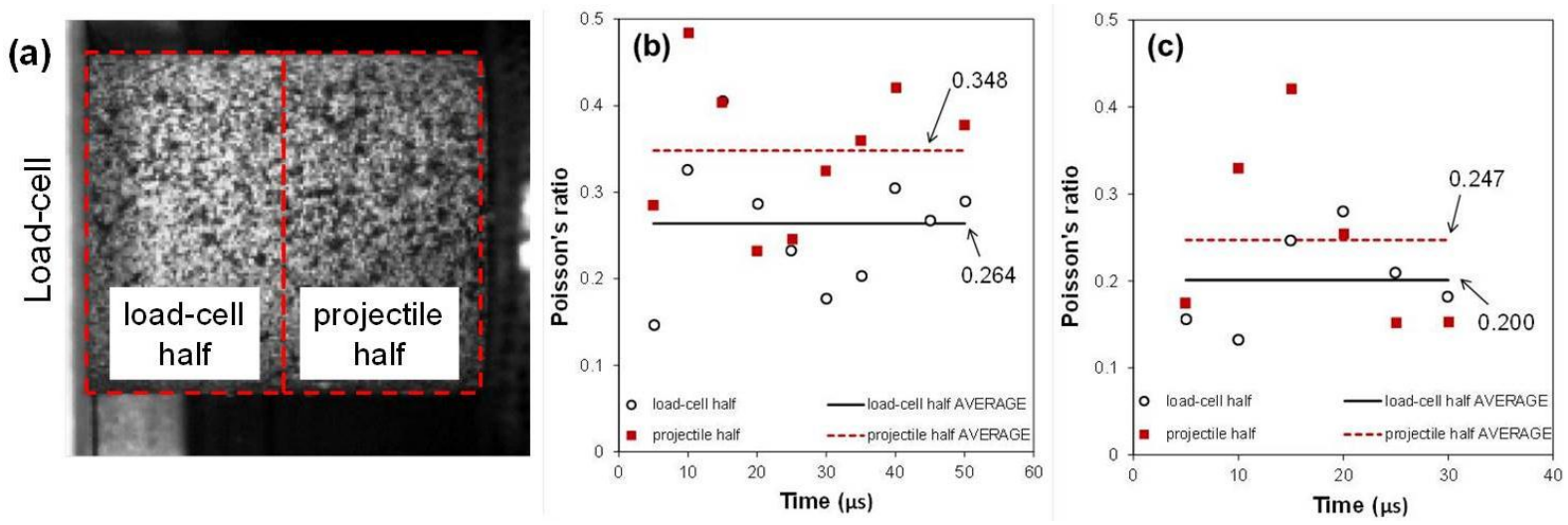

Fig. 8- Variation of Poisson's ratio with time extracted from the load-cell half and projectile half shown in (a), for (b) short and (c) long specimen.

Next, change of mass density over the entire area of interest is calculated using Eq. 3 and based on the average Poisson's ratios determined earlier. Fig. 9 depicts the variation of normalized density in time, for the same five locations shown earlier in Fig. 7a. Considering the density curves in Fig. 9, the following remarks are highlighted:

1) Local density increase of up to $11 \%$ is observed in both specimens.

2) There is a considerable delay in the rise time of curves extracted from $A_{1}$ and $A_{5}$ in both specimens. The delay times are approximately $8 \mu \mathrm{s}$ and $14 \mu \mathrm{s}$ for short and long specimens, respectively. These delays are due to the elapsed time for the stress wave to traverse the length of the specimen from impacted side to the load-cell side. Similar response was also observed earlier in Fig. 7.

3) Consistent with the full-field strain response, both specimens show appreciable spatial variation in density, whereas such spatial variability is more prominent in the long specimen. The short specimen shows maximum compressibility on its mid-length locations $\left(A_{2}\right.$ and $\left.A_{3}\right)$. This is because (i) the highest axial strains are developed within middle sections of this 
specimen (see Fig. 6a); (ii) lower Poisson's ratios are measured on the areas closer to the load-cell side of the specimen.

4) Although higher Poisson's ratio values are obtained over the projectile side in the long specimen, substantially higher local densities are determined on its impacted side. This is due to significantly larger axial plastic strains evidenced previously in Fig. 6 b.
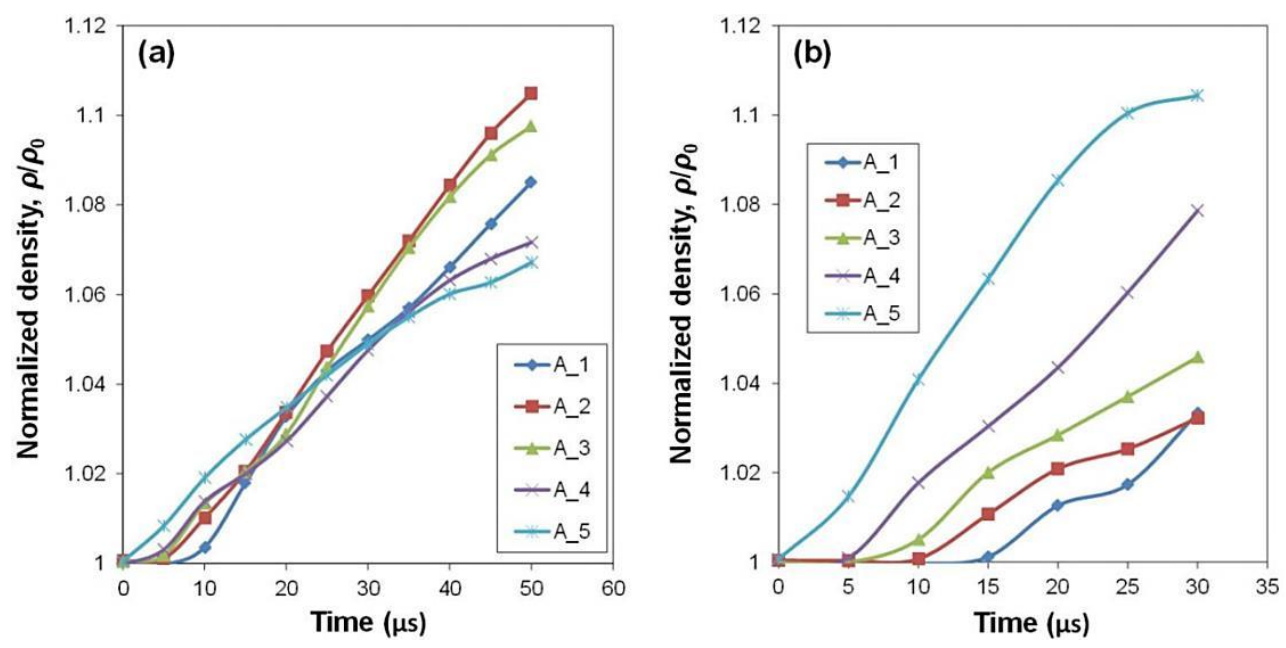

Fig. 9- Variation of normalized density, $\rho / \rho_{0}$, in time for (a) short and (b) long specimen, extracted for the representative locations indicated in Fig. 7a. $\left(\rho_{0}=560 \mathrm{~kg} / \mathrm{m}^{3}\right)$

\subsection{Strain Rate and Acceleration}

Distributions of temporal derivatives - strain rate and acceleration - are determined over the gauge area using the DIC displacement and strain data and by applying a central difference scheme:

$\dot{\varepsilon}_{i j}(t)=\frac{\varepsilon_{i j}(t+\Delta t)-\varepsilon_{i j}(t-\Delta t)}{2 \Delta t}$ 
$a_{i}(t)=\frac{u_{i}(t+\Delta t)-2 u_{i}(t)+u_{i}(t-\Delta t)}{\Delta t^{2}}$

where $u_{i}$ and $\varepsilon_{i j}$ denote the components of the displacement and strain, respectively. $a_{i}$ and $\dot{\varepsilon}_{i j}$ represent acceleration and strain rate, respectively. $\Delta t$ is inter-frame time $(=5 \mu \mathrm{s})$.

Variation of axial strain rate along the specimen axis has been plotted in Fig. 10. Distribution of axial strain rate in the short specimen is such that at $t<30 \mu \mathrm{s}$, the highest strain rate values are measured at the impacted side of the specimen. At $t>30 \mu \mathrm{s}$, the highest strain rate regions move towards the middle section of the specimen. Similar behavior is observed in the long specimen, as well. This behavior can be attributed to the plastic wave traverse along the specimen axis. As the plastic wave passes along the specimen from the impacted side, it reduces its velocity to zero, while plastically deforming the portion of the specimen left behind [36].

It is clearly indicated that at any given time during the deformation stage, there is a substantial variation in local strain rate values within the gauge area. Up to $4000 \mathrm{~s}^{-1}$ spatial variation in strain rate is observed in the specimens in this work, whereas such spatial variation is more prominent in the long specimen. This high variation in strain rate -along with the strong rate sensitivity of the examined material- can actually be turned into an advantage, since the ultimate goal here is to identify a rate-dependent constitutive law for the examined foam using a minimum number of dynamic experiments. More discussion on this subject can be found in the following sections. 

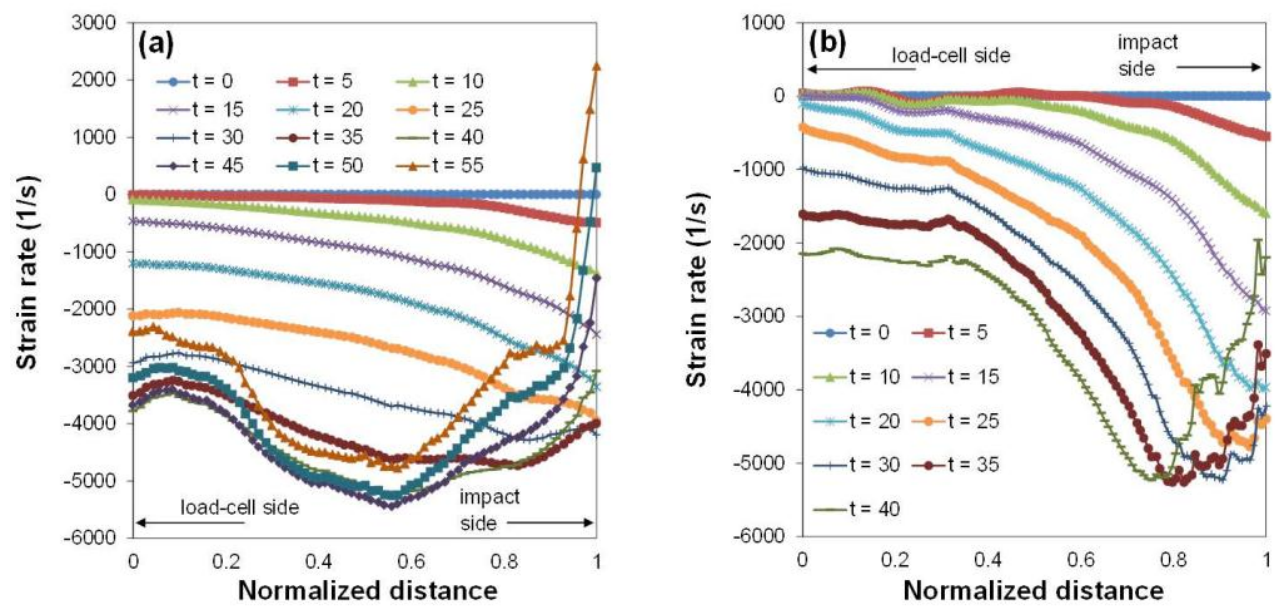

Fig. 10- Variation of axial strain rate along the specimen axis for (a) short and (b) long specimens.

Fig. 11 shows the variation of axial acceleration extracted from five locations along the specimen axis. Acceleration curves in Fig. 11 show strong length-wise gradients. In both specimen geometries, the axial acceleration curves show a significant drop during the first 25-30 $\mu$ s after the impact. The large magnitudes of acceleration calculated in early stages of impact attribute to the development of significant inertia stresses. After an early rapid increase in the magnitude of inertia stress, the acceleration is expected to be damped over a time duration that is roughly equal to 3 reverberations of the elastic stress wave. This is previously studied and confirmed in [31], where lower impact velocities were used to deform a similar material. In the current work, owing to the substantially higher impact velocities and the resultant applied strain rate, the long specimen fails before acceleration damping. In the short specimen, although deformation time is longer than that of the long specimen, complete damping does not take place and as a result, the effects of inertia stresses are significant and need to be included in the full-field stress analysis. 

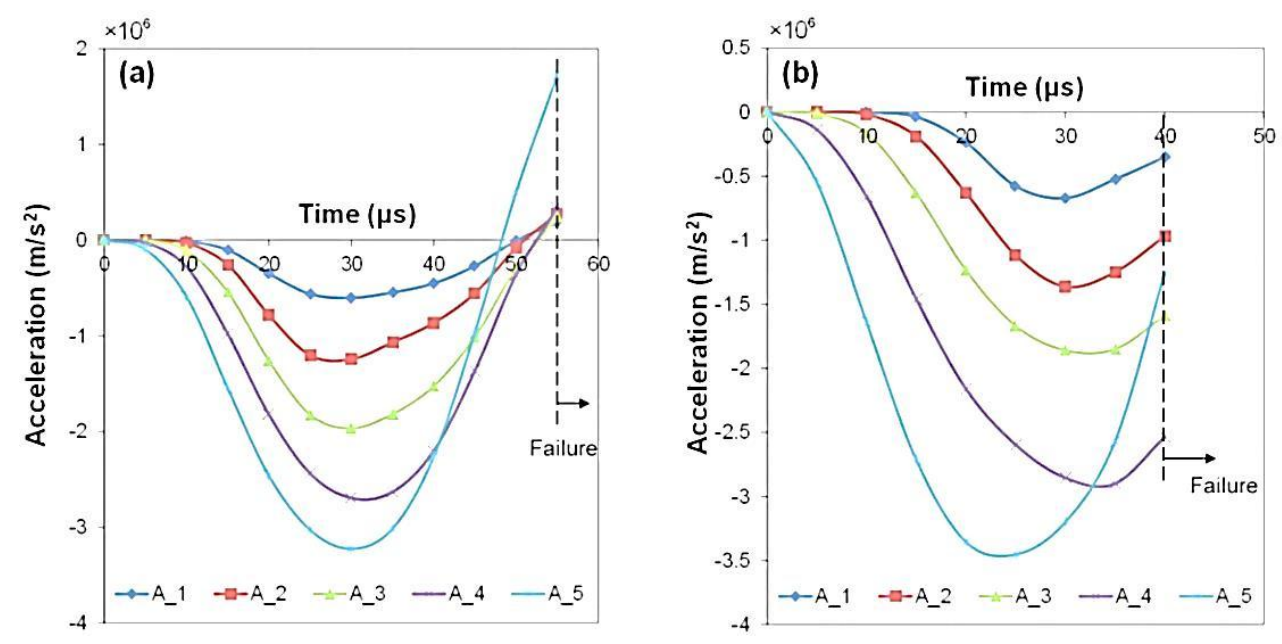

Fig. 11- Variation of axial acceleration with time for (a) short and (b) long specimen, extracted for the representative locations indicated in Fig. 7a.

\subsection{Inertia Stress and Full-Field Axial Stress}

Having obtained density and acceleration fields, inertia stress distribution over the entire area of interest can be evaluated for both specimens using Eqs. 2-4. Fig. 12 illustrates the evolution of inertia stresses along the axis of each specimen at different times prior to failure. Axial inertia stress developed in the specimen is clearly indicated to have the highest magnitude at the impacted side at any given time. In addition, higher values of inertia stress are measured in the long specimen, due to the higher impact velocity and the resultant higher axial accelerations. 

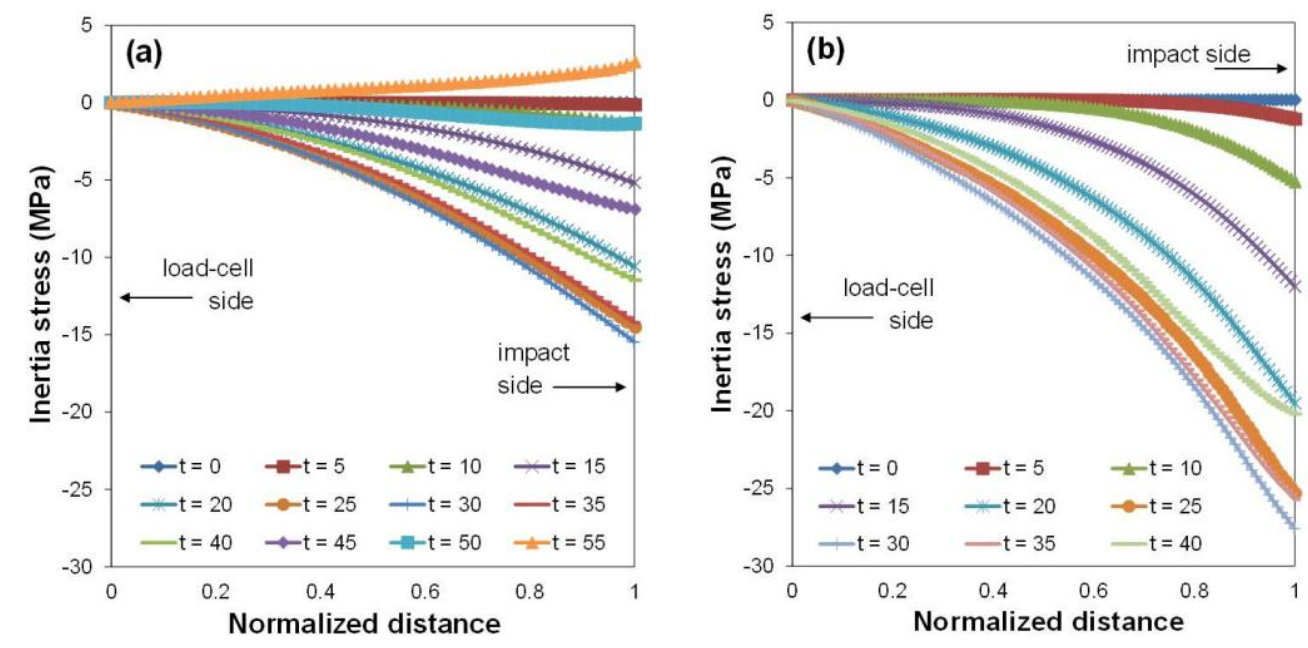

Fig. 12- Variation of inertia stress along the specimen axis for (a) short and (b) long specimens.

Variation of total axial stress along the specimen axis is obtained next by superimposing the inertia stress with the boundary measured stress (see Eq. 2). The true stress value at the boundary, i.e. load-cell side, is evaluated as the ratio between the reaction force measured by the load-cell and the instantaneous cross sectional area of the specimen. The instantaneous cross sectional area was determined from the 3D digital image correlation measurement of specimen profile facilitated by transforming the original Cartesian coordinate system into a polar coordinate system. This approach has been explained in more details in [31].”. Evolution of true stress at the load-cell side of the specimens is shown in Fig. 13. Note that the boundary measured stress is compressive, but has been plotted in positive values in this figure, for convenience. Both curves in Fig. 13 show progressive increase in stress until failure, and continue to rise even after the specimens fail. As mentioned earlier, specimen failure in this work is referred to the observation of visible surface cracks mostly formed on regions closer to the impacted side of each specimen, followed by total crushing. At the instant of failure, although the integrity of the specimen is lost, the stress wave can still 
propagate through the undamaged parts of the specimen. This attributes to the steady increase of the boundary measured force for at least $40 \mu \mathrm{s}$ after the failure.

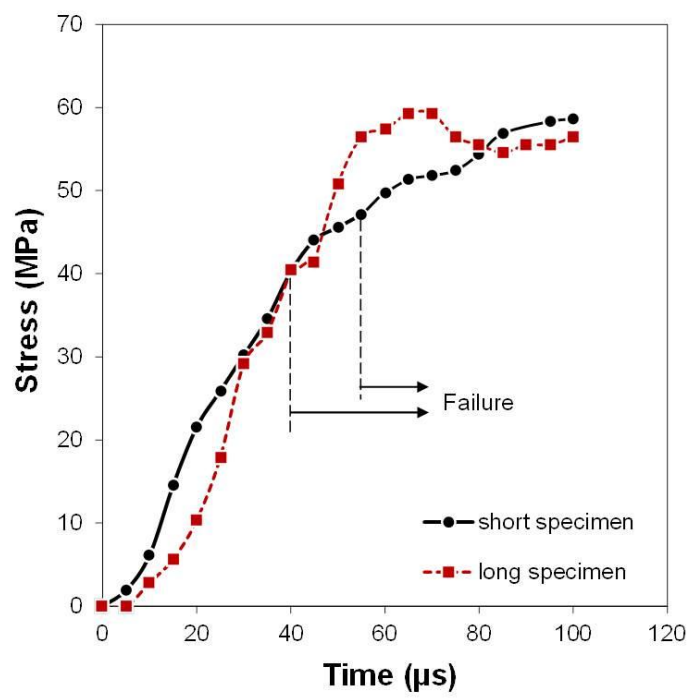

Fig. 13- Variation of true stress measured at the boundary for short and long specimens.

Variation of total, i.e. inertia plus boundary measured, axial stress along the specimen axis is plotted in Fig. 14 for short and long specimens. It should be noted that:

1) Total axial stress values indicate considerable length-wise variability, the degree of which is higher for the long specimen.

2) Magnitude of the total axial stress is larger at the impact side of the specimen, for the entire deformation time in the long specimen, as well as the first $45 \mu$ s after the impact in the short specimen.

3) At $t>45 \mu \mathrm{s}$, upon the reversion of the axial acceleration in the short specimen, the inertia stress changes sign. This was evidenced earlier in Figs. 11 and 12. The sign change gives rise to the development of tensile inertia stresses at the impacted side of the specimen, decreasing 
the magnitude of the previously developed compressive stresses and consequently lowering the spatial variation of stress along the specimen axis. Predominance of tensile inertia stresses over the areas closer to the impacted side of the specimen is indicated to promote tensile strain rates at $t>45 \mu$ s in the short specimen, as depicted earlier in Fig. 10a.
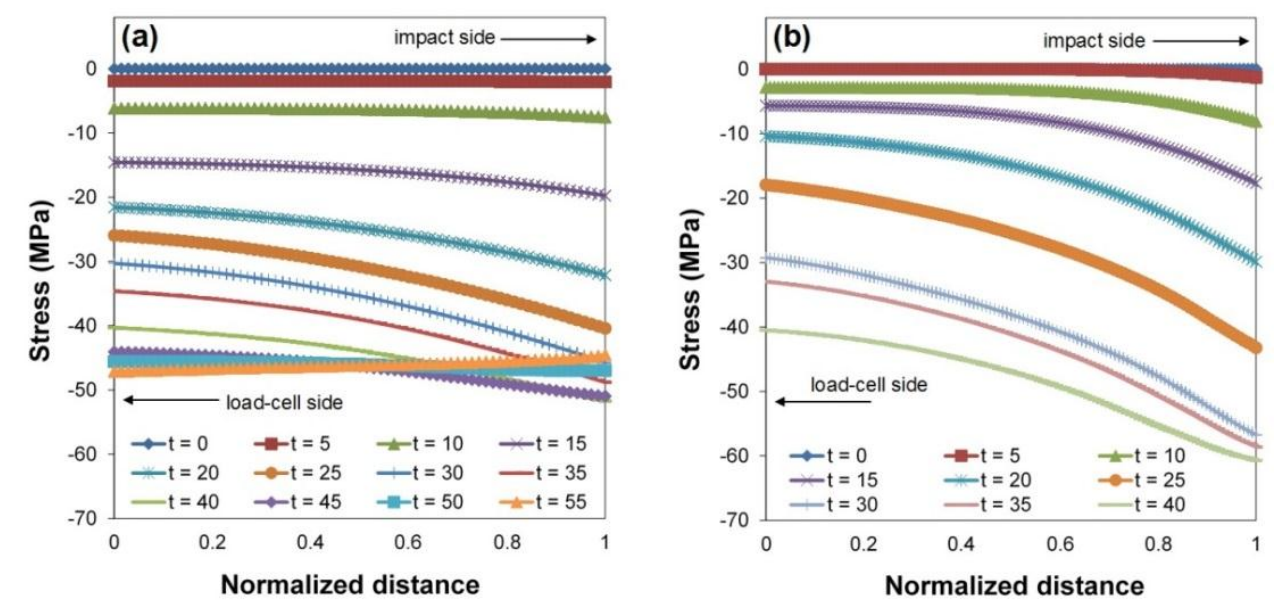

Fig. 14- Variation of total axial stress along the specimen axis for (a) short and (b) long specimens at different times after the impact.

\subsection{Identification of Inelastic Constitutive Response}

Fig. 15 shows the variation of strain, strain rate and total stress in time for both specimens. Note that all variables shown in Fig. 15 are compressive, however for convenience they are all plotted as positive quantities hereafter. The trends indicated for total stress are very similar to those observed in [31]. The only differences between the present result with those reported earlier are: (1) quasi-static equilibrium is not achieved before the specimen failure in this work; thus the stress-time curves do not tend to fully converge. This is particularly evident in stress-time curves obtained for the long specimen; (2) Due to the temporal filtering of the 
displacement data in the present work, no sudden increase is observed in the stress-time curves extracted from the impacted side; rather there exists a smoother rise in the value of total stress at earlier stages of deformation. In cases where no temporal smoothing is carried out, there will be a more abrupt increase of stress value on the locations more closely associated with the impacted side of the specimen [31].

(a)

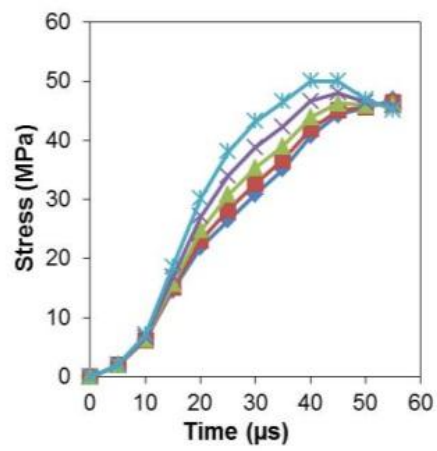

(b)

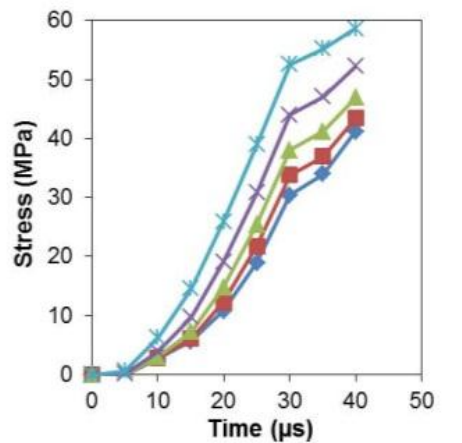

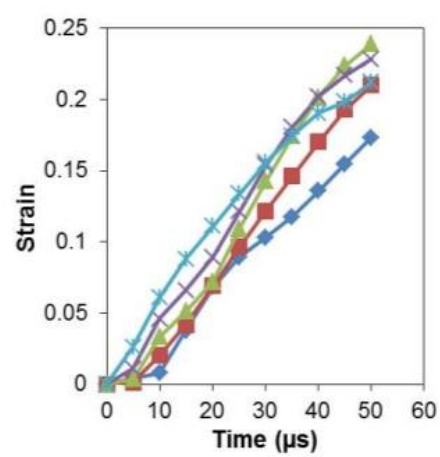
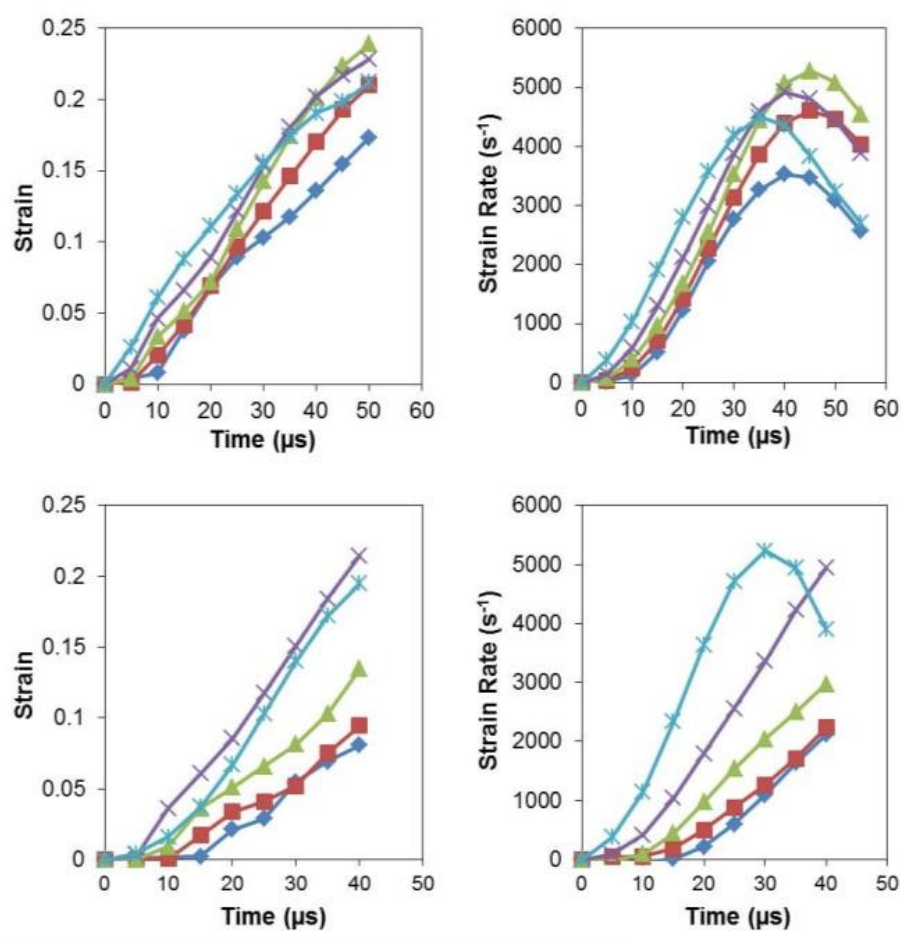

$\rightarrow$ A_1 $\rightarrow$-A_2 $\rightarrow$ A_3 $\div$-A_4 $\div$ - $\_5$

Fig. 15- Variation of total stress, strain and strain rate with time for (a) short and (b) long specimens extracted from the representative points indicated in Fig. 7a.

Having obtained the full-field strain, strain rate and stress distributions in time and over the entire gauge area, local stress-strain response of the material can be extracted at any given location within the area of interest. Accordingly, the local stress-strain curves extracted from three locations over the specimen axis are plotted in Fig. 16 along with their associated strain 
rate curves. It is clearly observed that although stress-strain curves can be acquired locally, no meaningful material response may be inferred from these local curves. This is because the local curves are not plotted at constant strain rates; not to mention that the issue becomes more challenging in the case of long specimen with higher spatial and temporal strain rate variabilities. High spatial variability of strain also attributes to the peculiar local stress-strain curves plotted in Fig. 16. This issue seems to be deteriorated for the longer specimen, containing higher strain inhomogeneity along its axis. Last but not least, strain and stress states vary in length of the specimen, changing from uniaxial strain state on the locations closer to the load-cell side to uniaxial stress state created behind the plastic wave front, over the locations closer to the impacted side. In this work we are only considering the axial stress-strain response of the material, regardless of the contribution of the lateral stresses and/or strains. Therefore, the discrepancies observed in Fig. 16 were actually not far from expectation.
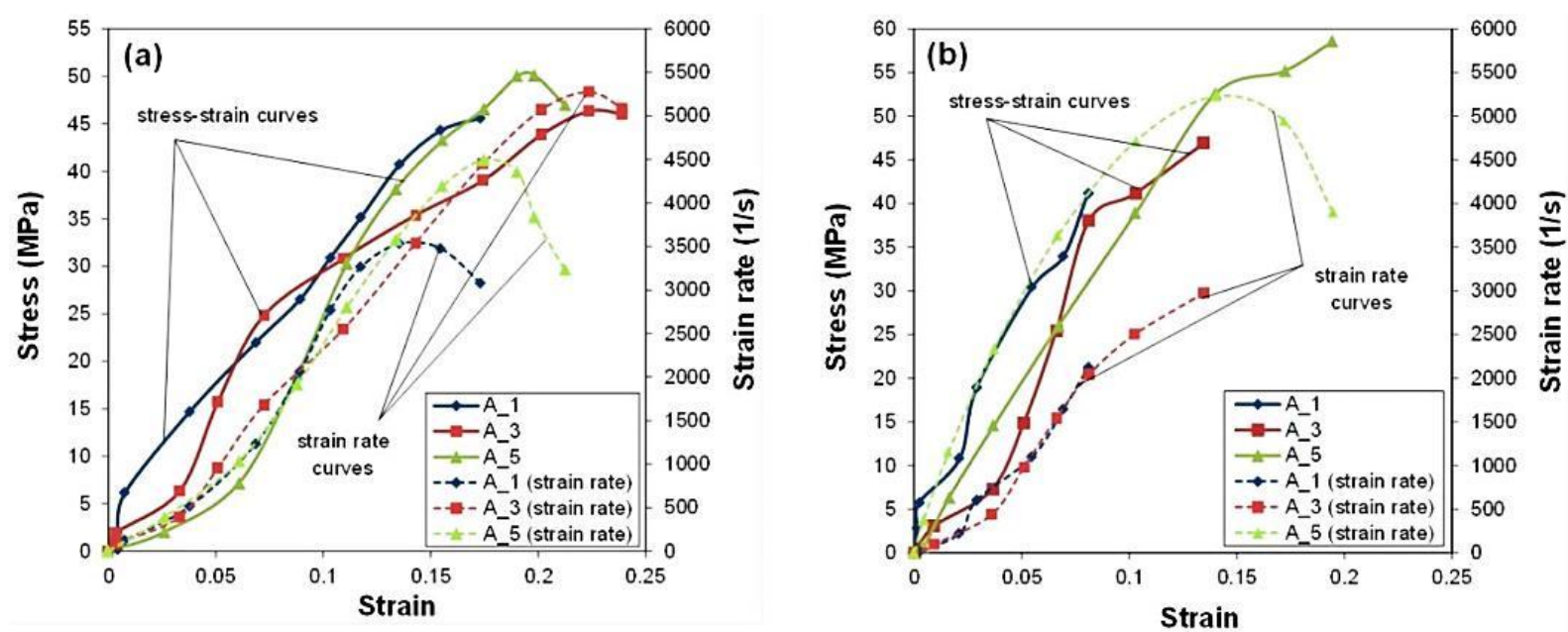

Fig. 16- Local stress-strain curves in (a) short and (b) long specimen, extracted for the representative locations indicated in Fig. 7a. 
To resolve the above-mentioned challenges and provide more meaningful constitutive information by obtaining stress-strain curves at constant strain rate conditions, the following mathematical approach is proposed:

1. Construct a full-grid material data set with known local strain, strain rate and stress data calculated within the gauge area. Note that the larger the number of data points in the grid, the more accurate the final results will be. In this work, the full-grid data set is constructed by combining the entire collection of stress-strain-strain rate data acquired for both short and long specimens.

2. Prescribing the target values for strain and strain rate, use a scattered-data interpolation scheme to estimate the local stress. In the present work, a triangulation-based cubic interpolation with $\mathrm{C}^{2}$ continuity is used for this purpose. The interpolation is performed in the software MATLAB ${ }^{\circledR}$, using an in-house written code.

3. Reconstruct stress-strain curves at a constant strain rate by repeating step 2 .

Following this procedure and using the entire collection of data points from both specimen geometries, constitutive curve of the material at constant strain rate of $1000 \mathrm{~s}^{-1}$ is first acquired over a strain range of 0.05 t0 0.125 , and set as the reference curve. Due to the limited strain data obtained within the strain rate range of $2000-5000 \mathrm{~s}^{-1}$, the curves corresponding to $\dot{\varepsilon}_{0}>1000 \mathrm{~s}^{-1}$ could not be directly reconstructed over the desired strain range of 0.05 to 0.125 . Therefore, setting the stress-strain curve interpolated for $\dot{\varepsilon}_{0}=1000 \mathrm{~s}^{-1}$ as the reference, and taking advantage of a rate dependent constitutive model, stress-strain curves at $\dot{\varepsilon}_{0}>1000 \mathrm{~s}^{-1}$ were identified for the examined foam. A simple rate-dependent constitutive model proposed by Nagy et al. [38] was considered for this purpose. The constitutive model suggested by Nagy et al. [38] is expressed as: 
$\sigma(\varepsilon)=\sigma_{0}(\varepsilon)\left(\frac{\dot{\varepsilon}}{\dot{\varepsilon}_{0}}\right)^{m(\varepsilon)}$

where $m(\varepsilon)$ is strain rate sensitivity exponent of the material, assumed to be strain dependent [38]. The reference stress, $\sigma_{0}$, in this work is taken to be the flow stress of the material at $\dot{\varepsilon}_{0}$ $=1000 \mathrm{~s}^{-1}$. Strain rate sensitivity of the material $m(\varepsilon)$ is determined using the reference curve (i.e. constitutive curve at $\dot{\varepsilon}_{0}=1000 \mathrm{~s}^{-1}$ ) and used to reconstruct stress-strain curves at $\dot{\varepsilon}_{0}>$ $1000 \mathrm{~s}^{-1}$.

Fig. 17a shows the variation of normalized stress vs. normalized strain rate at different strain magnitudes. The slope of the best linear fit for each curve is determined as the strain rate sensitivity. It is clearly observed that $m$ values calculated in the strain range of $1000 \mathrm{~s}^{-1}$ to $5000 \mathrm{~s}^{-1}$ indicate strong strain dependence. Fig. $17 \mathrm{~b}$ illustrates the variation of $m$ as a function of applied strain, with its best fitting curve. The trends observed for the straindependence of $m$ are similar to those previously documented in [38], confirming that the strain rate sensitivity increases at larger strains.

Fig. 18a illustrates the reconstructed flow curves at constant strain rates in the range of 1000 $\mathrm{s}^{-1}$ to $5000 \mathrm{~s}^{-1}$, confirming the more significant influence of strain rate on the constitutive response of the material at higher strain values. Please note that, the spatial and temporal variation of material density is already incorporated into the analysis from the beginning, by the utilizing a compressibility model described earlier in Eq.3

To further investigate the effect of strain rate on the constitutive behavior of the examined foam, stress values determined from the analytical approach are plotted as function of strain rate in Fig. 18b. Fig. 18b allows for a consistent comparison of strain rate effect on the stress response well beyond the elastic deformation regime and prior to failure. The trend observed 
in Fig. 18b indicates that beyond $1000 \mathrm{~s}^{-1}$, the foam exhibits a rapid increase in rate sensitivity. This observation is very similar to the previously documented data on various polymeric foam specimens $[39,40]$ and attributes to the critical strain rate hypothesis proposed for cellular structures [41].
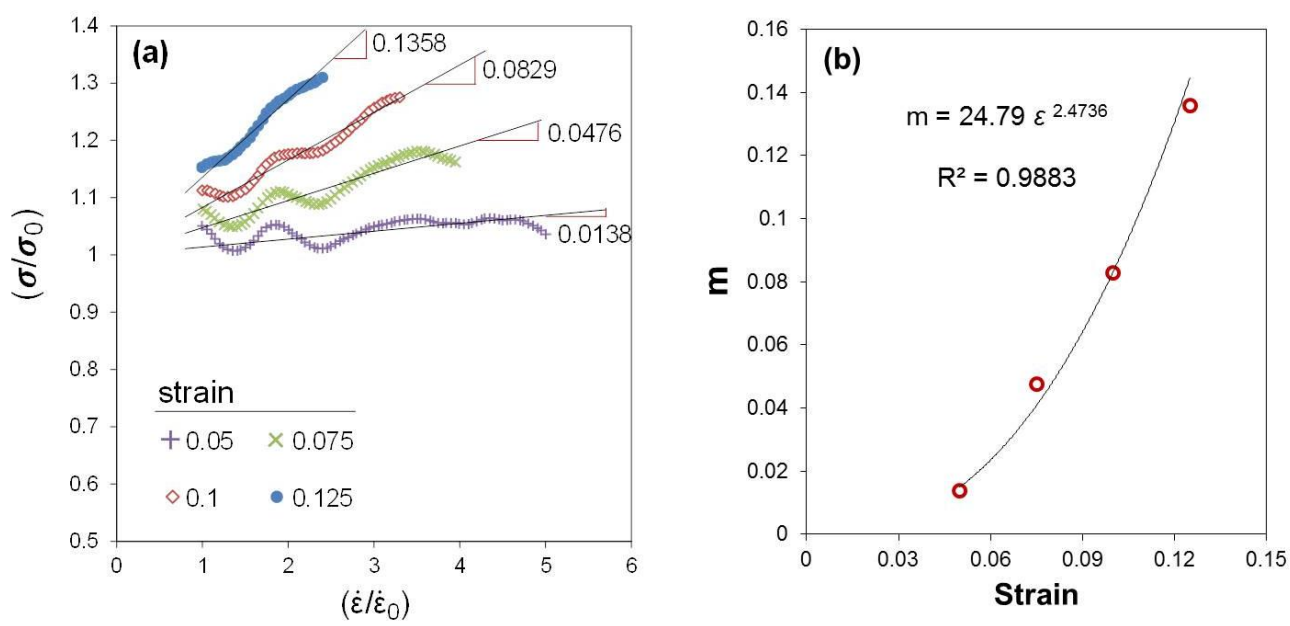

Fig. 17- Variations of (a) normalized stress vs. normalized strain rate at various strain magnitudes, and (b) strain rate sensitivity exponent, $m$, with strain over a range of $0.05<\varepsilon<0.125$ and $1000<\dot{\varepsilon}<5000 \mathrm{~s}^{-1}$.
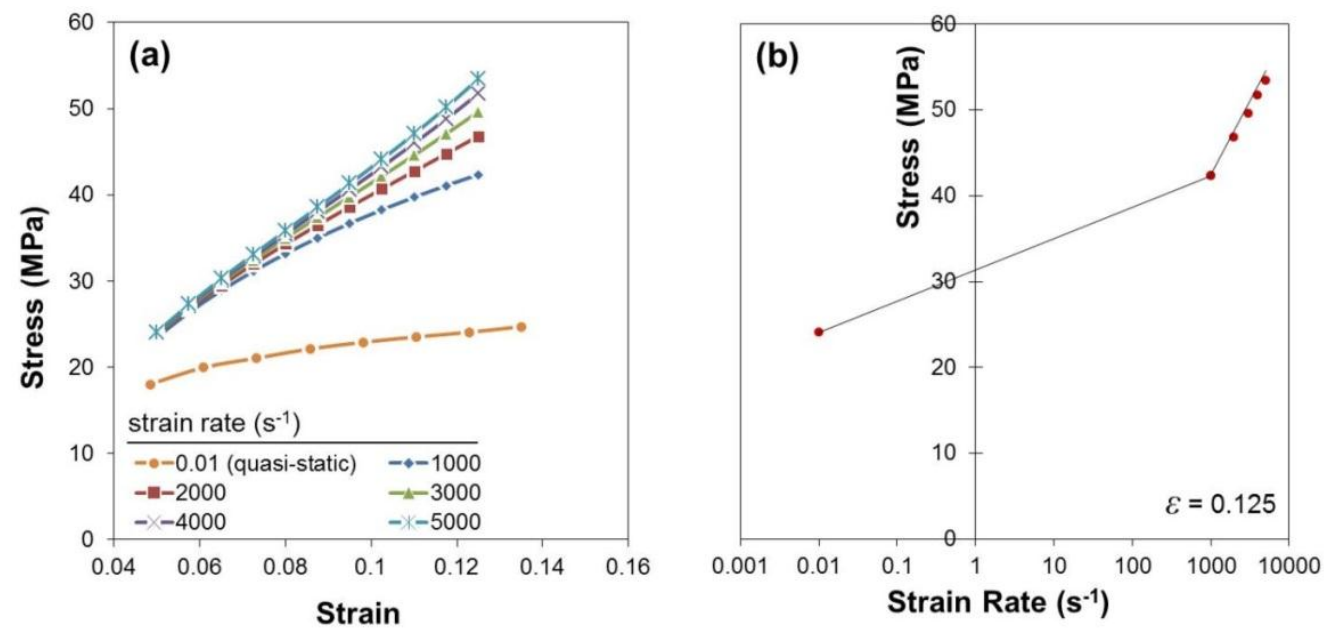
Fig. 18- (a) Stress-strain curves plotted for quasi-static and dynamic conditions. (b) Variation of stress with applied strain rate at a constant strain of 0.125 .

Finally, in order to validate the results obtained from this work, a comparison was made between the stress-strain response of the examined foam measured from conventional SHPB testing and the results acquired in this work. For this purpose, a conventional SHPB apparatus with details provided in [42] was employed. Smaller cubic specimens of $14 \times 14 \times 14 \mathrm{~mm}^{3}$ were used in SHPB tests to enforce more uniform deformation. Typical stress-strain and strain rate response obtained from SHPB tests on the specimen is shown in Fig. 19a. Note that the strain rate applied on the specimen in the SHPB test shows more temporal uniformity before failure. The average strain rate applied on the specimen over a strain range of 0.05 0.125 is determined as $1500 \mathrm{~s}^{-1}$ in the SHPB experiment. Using this average strain rate along with the results of the proposed analytical approach, the constitutive response of the material was predicted. Fig. 19b compares the constitutive response obtained from experimental SHPB and the model prediction. Note that the results obtained from the approach proposed in this work predict the non-linear stress-strain curve of the examined foam with $>90 \%$ accuracy. 

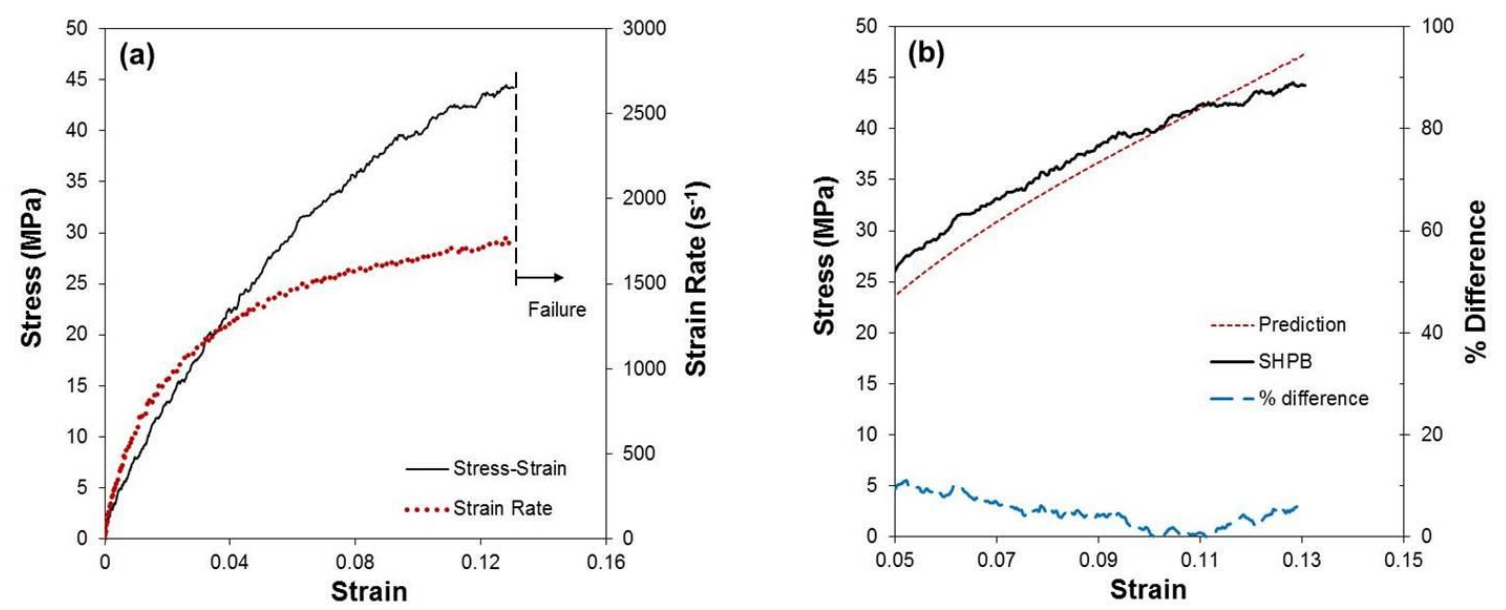

Fig. 19- (a) Constitutive response of the examined foam obtained from conventional SHPB.

(b) Comparison between the experimentally obtained and predicted stress-strain curves.

Last but not least, the approach described in this work can be considered as a novel methodology for extracting a large collection of data from a minimum number of testings. This method of analysis does not depend on any specific specimen geometry, nor does it require the use of any pulse shaping mechanism, since the effect of inertia loading is already embedded in the analysis. Clearly, this was the first attempt to demonstrate the feasibility of the application of the procedure detailed in this work. There are indeed several factors that can improve the results of the proposed approach. The most important factor is the application of more advanced cameras with enhanced temporal and spatial resolutions which allow for the acquisition of a larger number of higher resolution images at significantly shorter interframe times.

The parameter identification method presented in this work is still in its early phases, and certainly needs more improvement. One specific aspect which in our opinion can significantly improve the quality of the results of such approach is the use of more advanced mathematical tools to identify the trends hidden within the scattered data obtained from the 
non-parametric analysis. Simple scattered data interpolation was employed for this purpose in the present work. Attempts are currently being made to take advantage of more powerful mathematical tools, particularly neural networks [43], to further improve the results obtained from similar experiments.

\section{Conclusions}

A recently proposed non-parametric analysis was conducted to study the influence of specimen length-to-diameter ratio on constitutive response of polymeric foams under direct impact. The study was facilitated by the use of high speed stereovision photography in conjunction with 3D digital image correlation. Full-field stress distribution in the specimen was reconstructed by superimposing the boundary-measured stress with the calculated inertia stresses. Results obtained in this work clearly indicate that the effects of inertia are more significant in specimens with higher length-to-diameter ratios; however, taking advantage of the utilized non-parametric analysis, consistent constitutive behaviors can be obtained regardless of the specimen aspect ratio. One particular remark highlighted in the current study was that the approach implemented here facilitates the accurate characterization of the impact response of low impedance cellular polymers in conditions where stress equilibration does not take place before failure. Results obtained for the long specimen in this work are typical of such conditions, i.e. where the specimen failure occurs well before the establishment of quasi-static equilibrium.

Additionally, it was confirmed that the low impedance characteristic of the foam specimens can give rise to appreciable spatial variability in strain, strain rate and stress. In particular, temporal and spatial variations of strain rate elevate the complexities encountered in analyzing the constitutive response of the material. Spatial and temporal variabilities of strain rate were 
turned into an advantage, by conducting an analytical approach to identify the rate-dependent constitutive response of the material. It was finally proposed that that the method implemented in the present research can facilitate the identification of the viscoplastic constitutive laws over a wide range of strain rates, using minimum number of experimental testings.

\section{Acknowledgments}

Sandia National Laboratories is a multi-program laboratory managed and operated by Sandia Corporation, a wholly owned subsidiary of Lockheed Martin Corporation, for the U.S.

Department of Energy's National Nuclear Security Administration under contract DE-AC0494AL85000. 


\section{References}

[1] Gibson LJ, Ashby MF. Cellular solids: Structure and properties. $2^{\text {nd }}$ ed. Oxford. 1997.

[2] Zhao H. Testing of polymeric foams at high and medium strain rates. Polymer Testing 1997; 16: 507-516.

[3] Liu Q, Subhash G, Gao XL. A parametric study on crushability of open-cell structural polymeric foams. Journal of Porous Materials 2005; 12: 233-248.

[4] Liu Q, Subhash G. A phenomenological constitutive model for foams under large deformations. Polymer Engineering and Science 2004; 44(3): 463-473.

[5] Subhash G, Liu Q. Crushability maps for structural polymeric foams in uniaxial loading under rigid confinement. Experimental Mechanics 2004; 44(3): 289-294.

[6] Pal S, Maiti S, Subhash G. Effect of microscopic deformation mechanisms on the dynamic response of soft cellular materials. Mechanics of Materials 2010; 42: 118-133.

[7] Yunoshev AS, Sil'vestrov VV. Development of the polymeric split Hopkinson bar technique. Journal of Applied Mechanics and Technical Physics 2001; 42(3): 558-564.

[8] Casem DT, Fourney WL, Chang P. A polymeric split Hopkinson pressure bar instrumented with velocity gages. Experimental Mechanics 2003; 43(4): 420-427.

[9] Chen W, Zhang B, Forrestal MJ. A split Hopkinson bar technique for low-impedance materials. Experimental Mechanics 1999; 39(2): 81-85.

[10] Bacon C. An experimental method for considering dispersion and attenuation in a viscoelastic Hopkinson bar. Experimental Mechanics 1998; 38(4): 242-249. 
[11] Wang L, Labibes K, Azari A, Pluvinage G. Generalization of split Hopkinson bar technique to use viscoelastic bars. International Journal of Impact Engineering 1994; 15(5): 669-686.

[12] Zhao H, Gary G, Klepaczko JR. On the use of a viscoelastic split Hopkinson pressure bar. International Journal of Impact Engineering 1997; 19(4): 319-330.

[13] Sharma A, Shukla A, Prosser RA. Mechanical characterization of soft materials using high speed photography and split Hopkinson pressure bar technique. Journal of Materials Science 2002; 37(5): 1005-1017.

[14] Casem DT, Fourney W, Chang P. Wave separation in viscoelastic pressure bars using single-point measurements of strain and velocity. Polymer Testing 2003; 22(2): 155-164.

[15] Subhash G, Liu Q, Gao X. Quasistatic and high strain rate uniaxial compressive response of polymeric structural foams. International Journal of Impact Engineering 2006; 32(7): 1113-1126.

[16] Chen W, Lu F, Winfree N. High strain rate compressive behavior of a rigid polyurethane foam with various densities. Experimental Mechanics 2002; 42(1): 65-73.

[17] Song B, Chen W, Frew DJ. Dynamic compressive response and failure behavior of an epoxy syntactic foam. Journal of Composite Materials 2004; 38(11): 915-936.

[18] Gama BA, Lopatnikov SL, Gillespie JW. Hopkinson bar experimental technique: A critical review. Applied Mechanics Reviews 2004; 57(4): 223-250.

[19] Ouellet S, Cronin DS, Moulton J, Petel EO. High rate characterization of polymeric closed-cell foams: Challenges related to size effects. In: Chalivendra V, Song B, Casem D (Editors), Dynamic behavior of materials - Volume 1, Proceedings of the 2012 Annual 
Conference on Experimental and Applied Mechanics, Springer, 2013, pp 21-28. DOI 10.1007/978-1-4614-4238-7_4

[20] Kanaun S, Tkachenko O. Representative volume element and effective elastic properties of open cell foam materials with random microstructures. Journal of Mechanics of Materials and Structures. 2007; 2(7): 1607-1628.

[21] Alsayednoor J, Harrison P, Guo Z. Large strain compressive response of 2-D periodic representative volume element for random foam microstructures. Mechanics of Materials 2013; 66: 7-20.

[22] Liu J, Saletti D, Pattofatto S, Zhao H. Impact testing of polymer foam using Hopkinson bars and digital image analysis. Polymer Testing 2014; 36: 101-109.

[23] Sutton MA, Orteu JJ, Schreier HW. Image correlation for shape, motion and deformation measurements. Springer. NY. 2009

[24] Mallon S, Koohbor B, Kidane A, Sutton MA. Fracture behavior of prestressed composites subjected to shock loading: A DIC-based study. Experimental Mechanics 2015; 55: $211-225$.

[25] Gilat A, Schmidt TE, Walker AL. Full field measurement in compression and tensile split Hopkinson bar experiments. Experimental Mechanics 2009; 49: 291-302.

[26] Pierron F, Zhu H, Siviour C. Beyond Hopkinson's bar. Philosophical Transactions of the Royal Society A 2014; 372: 20130195.

[27] Moulart R, Pierron F, Hallett SR, Wisnom MR. Full-field strain measurement and identification of composite moduli at high strain rate with the virtual fields method. Experimental Mechanics 2011; 51: 509-536. 
[28] Yoon S, Giannakopoulos I, Siviour CR. Application of the virtual fields method to the uniaxial behavior of rubbers at medium strain rates. International Journal of Solids and Structures 2015; 69-70: 553-568.

[29] Othman R, Aloui S, Poitou A. Identification of non-homogeneous stress fields in dynamic experiments with a non-parametric method. Polymer Testing 2010; 29: 616-623.

[30] Koohbor B, Mallon S, Kidane A, Lu WY. The deformation and failure response of closed-cell PMDI foams subjected to dynamic impact loading. Polymer Testing 2015; 44: $112-124$.

[31] Koohbor B, Kidane A, Lu WY, Sutton MA. Investigation of the dynamic stress-strain response of compressible polymeric foam using a non-parametric analysis. International Journal of Impact Engineering 2016; 91: 170-182.

[32] Avril S, Pierron F, Sutton M, Yan J. Identification of elasto-visco-plastic parameters and characterization of Lüders behavior using digital image correlation and the virtual fields method. Mechanics of Materials 2008; 40: 729-742.

[33] Samanta SK. Dynamic deformation of aluminum and copper at elevated temperatures. Journal of Mechanics and Physics of Solids 1971; 19: 117.

[34] Wang L, Ding Y, Yang L. Experimental investigation on dynamic constitutive behavior of aluminum foams by new inverse methods from wave propagation measurements. International Journal of Impact Engineering 2013; 62: 48-59.

[35] Davies EDH, Hunter SC. The dynamic compression testing of solids by the method of the split Hopkinson pressure bar. Journal of Mechanics and Physics of Solids 1963; 11(3): $155-179$. 
[36] Nicholas T, Recht RF. Introduction to impact phenomena. In: High Velocity Impact Dynamics, Edited by Zukas JA. 1990, John Wiley \& Sons, 1990.

[37] Taylor G. The use of flat-ended projectiles for determining dynamic yield stress - I. Theoretical considerations. Philosophical Transactions of the Royal Society A 1948; 194: 289-299.

[38] Nagy A, Ko WL, Lindholm US. Mechanical behavior of foamed materials under dynamic compression. Journal of Cellular Plastics 1974; 10(3): 127-134.

[39] Ouellet S, Cronin D, Worswick M. Compressive response of polymeric foams under quasi-static, medium and high strain rate conditions. Polymer Testing 2006; 25: 731-743.

[40] Bouix R, Viot, Lataillade JL. Polypropylene foam behavior under dynamic loadings: Strain rate, density and microstructure effects. International Journal of Impact Engineering 2009; 36: 329-342.

[41] Tan PJ, Reid SR, Harrigan JJ. On the dynamic mechanical properties of open-cell metal foams - A re-assessment of the 'simple-shock theory'. International Journal of Solids and Structures 2012; 49: 2744-2753.

[42] Ravindran S, Tessema A, Kidane A. Local deformation and failure mechanisms of polymer bonded energetic materials subjected to high strain rate loading. Journal of Dynamic Behavior of Materials 2016; 2: 146-156.

[43] Bobbili R, Madhu V, Gogia AK. Neural network modeling to evaluate the dynamic flow stress of high strength armor steels under high strain rate compression. Defence Technology 2014; 10: 334-342. 


\section{Supplementary Material}

Video 1: The deformation and failure of the short specimen subjected to direct impact loading. (File name: short_specimen.mp4)

Video 2: The deformation and failure of the long specimen subjected to direct impact loading. (File name: long_specimen.mp4) 